\title{
SURFACES OF CONSTANT MEAN CURVATURE
}

\author{
JOSEPH A. WOLF ${ }^{1}$
}

1. Introduction. Let $S$ be a surface immersed in euclidean space $\boldsymbol{R}^{3}$ with constant mean curvature $H$. In a recent note [3] we proved that the quadratic differential form $-H I+I I$ is a flat Lorentz metric on the complement of the umbilic set of $S$. Here the result is used to set up a certain type of isothermal local coordinate system on $S$. The main consequences are:

(i) an obstruction theory, which tells one when an isometry of connected surfaces of the same constant mean curvature is a congruence; ${ }^{2}$

(ii) Gauss curvature on $S$ is set up as a solution to a nonlinear elliptic boundary value problem; and

(iii) construction of local surfaces of any given constant mean curvature.

2. Notation. $S$ denotes a surface with a fixed immersion $\nu: S \rightarrow R^{3}$. If $\xi$ is a smooth choice of unit normal defined over an open set $U \subset S$, then we recall the fundamental forms of the immersion:

$I=d \nu \cdot d \nu$, first fundamental form;

$I I=d \nu \cdot d \xi$, second fundamental form;

$I I I=d \xi \cdot d \xi$, third fundamental form.

$I=d \nu^{2}$ is the riemannian metric induced on $S$ by the immersion. The eigenvalues of $I I$ relative to $I$ are the principle curvatures, denoted $k_{i}$. As usual we have functions $H, K$ on $S$ given by

$$
\begin{aligned}
& H=\frac{1}{2}\left\{k_{1}+k_{2}\right\}, \text { mean curvature; } \\
& K=k_{1} k_{2}, \text { Gauss curvature. }
\end{aligned}
$$

They define the quadratic differential form

$$
\Omega=-H I+I I, \text { modified fundamental form. }
$$

The eigenvalues of $\Omega$ relative to $I$ are $k_{i}-\frac{1}{2}\left(k_{1}+k_{2}\right)= \pm \frac{1}{2}\left(k_{1}-k_{2}\right)$. Thus $\Omega$ is a pseudo-riemannian metric of Lorentz signature (Lorentz metric) on the open subset

$$
S_{\Omega}=\left\{x \in S: k_{1}(x) \neq k_{2}(x)\right\}
$$

Received by the editors January 16, 1966.

${ }^{1}$ Research supported in part by National Science Foundation Grant GP2439, in part by an Alfred P. Sloan Research Fellowship.

2 In other words, when the isometry is the restriction of a rigid motion of the ambient euclidean space $\boldsymbol{R}^{3}$. 
of $S$. We view $S_{\Omega}$ as a Lorentz surface with metric $\Omega$. Recall that a point $x \in S$ is called umbilic if $k_{1}(x)=k_{2}(x)$; thus $S_{\Omega}$ is the complement of the umbilic set of $S$.

3. Special coordinates on $S_{\Omega}$. The results of this note are based on the following observation.

3.1. Theorem. Let $S$ be a surface immersed in $R^{3}$ with constant mean curvature $H$. Let $K$ denote Gauss curvature and define $e^{3}$ a function

$$
\lambda=-\frac{1}{2} \log \left(H^{2}-K\right) \text { on } S_{\Omega} .
$$

If $x \in S_{\Omega}$, then $x$ has a local coordinate neighborhood ${ }^{4}(U, u)$ with $U \subset S_{\Omega}$ and

$$
\begin{aligned}
I & =e^{\lambda}\left\{d u^{1} \otimes d u^{1}+d u^{2} \otimes d u^{2}\right\} \\
I I & =\left(H e^{\lambda}+1\right) d u^{1} \otimes d u^{1}+\left(H e^{\lambda}-1\right) d u^{2} \otimes d u^{2} \\
k_{1} & =H+e^{-\lambda}, \quad k_{2}=H-e^{-\lambda}, \quad K=H^{2}-e^{-2 \lambda} .
\end{aligned}
$$

If $(V, v)$ is another local coordinate neighborhood of $x$ with these properties, then $v^{i}= \pm u^{i}+c^{i}, c^{i}$ constant, on each component of $U \cap V$.

PROOF. Let the principle curvature be numbered so that $k_{1}>k_{2}$ on $S_{\Omega}$. Given $x \in S_{\Omega}$ we choose a neighborhood $W \subset S_{\Omega}$ of $x$ which carries an $I$-orthonormal moving frame $\left\{X_{1}, X_{2}\right\}$ such that $X_{i}$ is a principle vector with principle curvature $k_{i}$. We have seen [3, Corollary 4.11] that the connection form of the Lorentz surface $S_{\Omega}$ is identically zero in the $\Omega$-orthonormal moving frame $\left\{Y_{1}, Y_{2}\right\}$, where $Y_{i}=\left\{\frac{1}{2}\left(k_{1}-k_{2}\right)\right\}^{1 / 2} X_{i}$. It follows that $x$ has a local coordinate neighborhood $(U, u)$ such that $U \subset W$ and $\partial / \partial u^{i}=Y_{i}$. Now

$$
\Omega=d u^{1} \otimes d u^{1}-d u^{2} \otimes d u^{2} \text { in } U .
$$

On the other hand, $I$ and $I I$ are diagonalized by $\left\{X_{1}, X_{2}\right\}$, hence also by $\left\{Y_{1}, Y_{2}\right\}=\left\{\partial / \partial u^{1}, \partial / \partial u^{2}\right\}$. Thus

$$
I=\sum_{1}^{2} g_{i} d u^{i} \otimes d u^{i} \quad \text { and } \quad I I=\sum_{1}^{2} b_{i} d u^{i} \otimes d u^{i}
$$

in $U$. This tells us

$$
b_{i}=k_{i} g_{i}, \quad-H g_{1}+b_{1}=1, \quad-H g_{2}+b_{2}=-1 .
$$

We compute

${ }^{3}$ Here we must observe that $H^{2}-K>0$ on $S_{\Omega}$; for $H^{2}-K=\frac{1}{4}\left(k_{1}-k_{2}\right)^{2}$.

${ }^{4} U$ is the neighborhood and $u=\left(u^{1}, u^{2}\right)$ is the local coordinate. 


$$
2 H=k_{1}+k_{2}=\frac{b_{1}}{g_{1}}+\frac{b_{2}}{g_{2}}=\frac{H g_{1}+1}{g_{1}}+\frac{H g_{2}-1}{g_{2}}=2 H+\frac{1}{g_{1}}-\frac{1}{g_{2}} .
$$

Thus $g_{1}=g_{2}$, which must be positive because $I$ is positive definite. Now $g_{1}=g_{2}=e^{\lambda}$ for some function $\lambda$ defined on $U$. We compute

$$
\begin{aligned}
& b_{1}=H g_{1}+1=H e^{\lambda}+1, \quad k_{1}=b_{1} / g_{1}=H+e^{-\lambda} ; \\
& b_{2}=H g_{2}-1=H e^{\lambda}-1, \quad k_{2}=b_{2} / g_{2}=H-e^{-\lambda} ; \\
& K=k_{1} k_{2}=H^{2}-e^{-2 \lambda}, \quad \text { so } \quad \lambda=-\frac{1}{2} \log \left(H^{2}-K\right) .
\end{aligned}
$$

This proves (3.3), (3.4) and (3.5).

For the uniqueness, observe that $\left\{\partial / \partial v^{1}, \partial / \partial v^{2}\right\}$ diagonalizes $I$ and $I I$ with first coefficient greater than second in $I I$. Thus $\partial / \partial v^{i}$ is a principle vector with principle curvature $k_{i}$ on $S$. As $\Omega\left(\partial / \partial v^{i}, \partial / \partial v^{i}\right)$ $=\Omega\left(\partial / \partial u^{i}, \partial / \partial u^{i}\right)= \pm 1 \neq 0$, now $\partial / \partial v^{i}= \pm \partial / \partial u^{i}$, so $d v^{i}= \pm d u^{i}$. q.e.d.

4. The Mainardi-Codazzi equations. Let $(U, u)$ be a connected local coordinate neighborhood on a surface $S$ immersed in $R^{\text {. Sup- }}$ pose that the fundamental forms are given by

(4.1) $I=e^{\lambda}\left\{d u^{1} \otimes d u^{1}+d u^{2} \otimes d u^{2}\right\}$ and $I I=\sum_{i j} b_{i j} d u^{i} \otimes d u^{j}$.

Then the Christoffel symbols are easily computed:

(4.2) $\Gamma_{11}^{1}=-\Gamma_{22}^{1}=\Gamma_{12}^{2}=\frac{1}{2} \frac{\partial \lambda}{\partial u^{1}} ; \Gamma_{12}^{1}=-\Gamma_{11}^{2}=\Gamma_{22}^{2}=\frac{1}{2} \frac{\partial \lambda}{\partial u^{2}}$.

Thus the Mainardi-Codazzi equations reduce to

$$
\begin{aligned}
& \frac{\partial b_{11}}{\partial u^{2}}-\frac{\partial b_{12}}{\partial u^{1}}=\frac{1}{2}\left(b_{11}+b_{22}\right) \frac{\partial \lambda}{\partial u^{2}} \text { and } \\
& \frac{\partial b_{22}}{\partial u^{1}}-\frac{\partial b_{12}}{\partial u^{2}}=\frac{1}{2}\left(b_{11}+b_{12}\right) \frac{\partial \lambda}{\partial u^{1}} .
\end{aligned}
$$

Now suppose that our surface $S$ has constant mean curvature $H$. Let $z=u^{1}+(-1)^{1 / 2} u^{2}$, complex local coordinate, and define

$$
\phi(z)=\left(b_{11}-b_{22}\right)+2(-1)^{1 / 2} b_{12} .
$$

As $2 H=b_{11} e^{-\lambda}+b_{22} e^{-\lambda}=\left(b_{11}+b_{22}\right) e^{-\lambda}$ is constant, (4.3) says that $\partial / \partial \bar{z}$ $=\frac{1}{2}\left\{\partial / \partial u^{1}+(-1)^{1 / 2} \partial / \partial u^{2}\right\}$ annihilates $\phi$; thus $\phi$ is a holomorphic function of $z$. Let $f$ be the function on $U$ defined by

$$
b_{11}=H e^{\lambda}+f, \quad b_{22}=H e^{\lambda}-f .
$$

Suppose that Gauss curvature satisfies 


$$
K=H^{2}-e^{-2 \lambda} \text {, i.e., } \lambda=-\frac{1}{2} \log \left(H^{2}-K\right) .
$$

Then

$$
H^{2} e^{2 \lambda}-1=K e^{2 \lambda}=b_{11} b_{22}-b_{12}^{2}=H^{2} e^{2 \lambda}-\left(f^{2}+b_{12}^{2}\right),
$$

so $f^{2}+b_{12}^{2}=1$. But $\phi=2\left(f+(-1)^{1 / 2} b_{12}\right)$ is holomorphic; now the maximum modulus principle says that $\phi$ is constant; thus $f$ and $b_{12}$ are constant.

Notice that $U \subset S_{\Omega}$ by the assumption $H^{2}-K=e^{-2 \lambda}>0$. Cutting $U$ down if necessary, Theorem 3.1 gives us a local coordinate $v$ on $U$ in which $I=e^{\lambda}\left\{d v^{1} \otimes d v^{1}+d v^{2} \otimes d v^{2}\right\}$ and $I I=\left(H e^{\lambda}+1\right) d v^{1} \otimes d v^{1}$ $+\left(H e^{\lambda}-1\right) d v^{2} \otimes d v^{2}$. If $\alpha$ is the oriented angle from $\partial / \partial u^{1}$ to $\partial / \partial v^{1}$, the two expressions for $I$ give

$d v^{1}=\cos \alpha d u^{1}+\sin \alpha d u^{2} \quad$ and $\quad d v^{2}=-\sin \alpha d u^{1}+\cos \alpha d u^{2}$.

Equating coefficients of $d u^{1} \otimes d u^{1}$ in the two expressions for $I I$,

$$
\begin{aligned}
H e^{\lambda}+f & =b_{11}=\left(H e^{\lambda}+1\right) \cos ^{2} \alpha+\left(H e^{\lambda}-1\right) \sin ^{2} \alpha \\
& =H e^{\lambda}+\left\{\cos ^{2} \alpha-\sin ^{2} \alpha\right\} .
\end{aligned}
$$

Thus $f=\cos ^{2} \alpha-\sin ^{2} \alpha=\cos (2 \alpha)$. Similarly $b_{12}=2 \cos \alpha \sin \alpha=\sin (2 \alpha)$. Now $\alpha$ is constant, and $v^{1}=\cos \alpha u^{1}+\sin \alpha u^{2}+c^{1}$ and $v^{2}=-\sin \alpha u^{1}$ $+\cos \alpha u^{2}+c^{2}$ for some constants $c^{i}$. We summarize as follows.

4.4. Theorem. Let $S$ be a surface immersed in $R^{3}$ with constant mean curvature $H$, and define $\lambda=-\frac{1}{2} \log \left(H^{2}-K\right)$ on $S_{\Omega}$. Let $(U, u)$ be a connected local coordinate neighborhood such that $U \subset S_{\Omega}$ and $I=e^{\lambda}\left\{d u^{1} \otimes d u^{1}+d u^{2} \otimes d u^{2}\right\}$. Then there is a constant $\alpha$ such that

$$
\begin{aligned}
I I= & \left(H e^{\lambda}+\cos 2 \alpha\right) d u^{1} \otimes d u^{1}+2 \sin 2 \alpha d u^{1} d u^{2} \\
& +\left(H e^{\lambda}-\cos 2 \alpha\right) d u^{2} \otimes d u^{2} .
\end{aligned}
$$

Let $c^{i}$ be constants, $v^{1}=\cos \alpha u^{1}+\sin \alpha u^{2}+c^{1}$ and $v^{2}=-\sin \alpha u^{1}+\cos \alpha u^{2}$ $+c^{2}$. Then $v=\left(v^{1}, v^{2}\right)$ is a local coordinate on $U, \alpha$ is the angle from $\partial / \partial u^{1}$ to $\partial / \partial v^{1}$, and

$$
\begin{aligned}
I & =e^{\lambda}\left\{d v^{1} \otimes d v^{1}+d v^{2} \otimes d v^{2}\right\} \quad \text { and } \\
I I & =\left(H e^{\lambda}+1\right) d v^{1} \otimes d v^{1}+\left(H e^{\lambda}-1\right) d v^{2} \otimes d v^{2} .
\end{aligned}
$$

5. Obstruction to a congruence. The following result generalizes the fact that an isometry of small patches of a right circular cylinder is a congruence only when it preserves the direction of the axis of the cylinder.

5.1. Theorem. Let $S, S^{\prime}$ and $S^{\prime \prime}$ be connected surfaces embedded in 
$R^{3}$ with the same constant mean curvature $H$, which are not open subsets of a plane or a sphere. Then to every isometry $f: S \rightarrow S^{\prime}$ we have a real number $\alpha(f)$, defined up to addition of an integral multiple of $\pi$, specified by the property: if $x \in S_{\Omega}$ and $(U, u)$ is a local coordinate neighborhood of $x$ given by Theorem 3.1 then $f^{*} I I^{\prime}=\left\{H e^{\lambda}+\cos 2 \alpha(f)\right\} d u^{1} \otimes d u^{1}$ $+2 \sin 2 \alpha(f) d u^{1} d u^{2}+\left\{H e^{\lambda}-\cos 2 \alpha(f)\right\} d u^{2} \otimes d u^{2}$ in $U$.

$\alpha$ has the properties:

(i) $f$ extends to a rigid motion of $R^{3}$, if and only if $\alpha(f) \equiv 0 \bmod \pi$;

(ii) $\alpha\left(f^{-1}\right)=-\alpha(f)$;

(iii) if $g: S^{\prime} \rightarrow S^{\prime \prime}$ is an isometry, then $\alpha(g \cdot f)=\alpha(f)+\alpha(g)$;

(iv) two isometries $f, g: S \rightarrow S^{\prime}$ differ by a rigid motion of $\boldsymbol{R}^{3}$, if and only if $\alpha(f) \equiv \alpha(g) \bmod \pi$.

Given $x \in S_{\Omega}$ and a real number $b$, there is a neighborhood $V$ of $x$, a surface $W$ of constant mean curvature $H$, and an isometry $h: V \rightarrow W$, such that $\alpha(h)=b$.

The proof is based on the standard fact [2]:

5.2. Lemma. Let $S$ be a connected surface immersed in $R^{3}$ with constant mean curvature. If some point of $S$ is not umbilic, then the umbilics of $S$ are isolated.

The function $\phi$ of $\$ 4$ is holomorphic, and the umbilics of $S$ in the domain of $\phi$ are just the zeroes of $\phi$.

Now the surfaces $S, S^{\prime}$ and $S^{\prime \prime}$ have all umbilics isolated; for an all-umbilic surface is an open subset of a plane or a sphere. Thus $S_{\Omega}$ (resp. $S_{\Omega}^{\prime}$, resp. $S_{\Omega}^{\prime \prime}$ ) is arcwise connected and dense in $S$ (resp. $S^{\prime}$, resp. $\left.S^{\prime \prime}\right)$. Let $K, K^{\prime}$ and $K^{\prime \prime}$ denote their Gauss curvature functions and let $f: S \rightarrow S^{\prime}$ be an isometry. Then $f\left(S_{\Omega}\right)=S_{\Omega}^{\prime}$ because $K^{\prime}(f(x))$ $=K(x), S_{\Omega}=\left\{x \in S: H^{2}-K(x)>0\right\}$ and $S_{\Omega}^{\prime}=\left\{f(x) \in S^{\prime}: H^{2}-K^{\prime}(f(x))\right.$ $>0\}$. Similarly, the functions $\lambda=-\frac{1}{2} \log \left(H^{2}-K\right)$ on $S_{\Omega}$ and $\lambda^{\prime}$ $=-\frac{1}{2} \log \left(H^{2}-K^{\prime}\right)$ on $S_{\Omega}^{\prime}$ are related by $\lambda=\lambda^{\prime} \cdot f$.

Let $x \in S_{\Omega}$ and choose a connected local coordinate neighborhood $(U, u)$ according to Theorem 3.1. Then $I=e^{\lambda}\left\{d u^{1} \otimes d u^{1}+d u^{2} \otimes d u^{2}\right\}$ and $I I=\left(H e^{\lambda}+1\right) d u^{1} \otimes d u^{1}+\left(H e^{\lambda}-1\right) d u^{2} \otimes d u^{2}$. Let $W=f(U), w^{i}(f(z))$ $=u^{i}(z)$ for $z \in U$; then $(W, w)$ is a connected local coordinate neighborhood of $f(x)$. $U \subset S_{\Omega}$ implies $W \subset S_{\Omega_{2}}^{\prime} \cdot f^{*} I^{\prime}=I$ because $f$ is an isometry, so $f^{*} I^{\prime}=e^{\lambda}\left\{d u^{1} \otimes d u^{1}+d u^{2} \otimes d u^{2}\right\}=e^{\lambda^{\prime} \cdot f}\left\{d\left(w^{1} \cdot f\right) \otimes d\left(w^{1} \cdot f\right)\right.$ $\left.+d\left(w^{2} \cdot f\right) \otimes d\left(w^{2} \cdot f\right)\right\}$; thus $I^{\prime}=e^{\lambda^{\prime}}\left\{d w^{1} \otimes d w^{1}+d w^{2} \otimes d w^{2}\right\}$. Applying Theorem 4.4 to $S^{\prime}$, we have a number $\alpha_{f}(x)$ such that $\boldsymbol{I I}^{\prime}=$ $\left.H e^{\lambda^{\prime}}+\cos 2 \alpha_{f}(x)\right) d w^{1} \otimes d w^{1}+2 \sin 2 \alpha_{f}(x) d w^{1} d w^{2}+\left(H e^{\lambda^{\prime}}-\cos 2 \alpha_{f}(x)\right) d w^{2}$ $\otimes d w^{2}$. Thus $f^{*} I I^{\prime}=\left(H e^{\lambda}+\cos 2 \alpha_{f}(x)\right) d u^{1} \otimes d u^{1}+2 \sin 2 \alpha_{f}(x) d u^{1} d u^{2}$ $+\left(H e^{\lambda}-\cos 2 \alpha_{f}(x)\right) d u^{2} \otimes d u^{2}$. This specifies $\alpha_{f}(x)$ up to an integral 
multiple of $\pi$. The uniqueness part of Theorem 3.1 says that $\alpha_{f}(x)$ is well defined up to an integral multiple of $\pi$.

Let $C$ be the circle which is the real numbers modulo $\pi$. We have a map $\alpha_{f}: S_{\Omega} \rightarrow C$. If $x \in S_{\Omega}$ then $\alpha_{f}$ is constant on a neighborhood of $x$. As $S_{\Omega}$ is connected, now $\alpha_{f}$ is constant. Let $\alpha(f)$ denote its value. We have proved the existence of a number $\alpha(f)$ defined modulo $\pi$ and specified by $f^{*} I I^{\prime}$ as required.

If $\alpha(f) \equiv 0 \bmod \pi$ if and only if $\cos 2 \alpha(f)=1$ and $\sin 2 \alpha(f)=0$, which is equivalent to $f^{*} I I^{\prime}=I I$. In that case $f: S \rightarrow S^{\prime}$ is a diffeomorphism of connected surfaces in $R^{3}$ such that $f^{*} I^{\prime}=I$ and $f^{*} I I^{\prime}=I I$, and a classical theorem says that $f$ extends to a rigid motion of $R^{3}$. This proves (i).

For (iii) we have a local coordinate $v$ on $W \subset S^{\prime}$ given by $\left(\alpha_{f}=\alpha(f)\right)$ $v^{1}=\cos \left(\alpha_{f}\right) w^{1}+\sin \left(\alpha_{f}\right) w^{2}$ and $v^{2}=-\sin \left(\alpha_{f}\right) w^{1}+\cos \left(\alpha_{f}\right) w^{2}$, and $I I^{\prime}$ $=\left(H e^{\lambda^{\prime}}+1\right) d v^{1} \otimes d v^{1}+\left(H e^{\lambda^{\prime}}-1\right) d v^{2} \otimes d v^{2}$ on $W$. Let $X=g(W) \subset S_{\Omega}^{\prime \prime}$ and let $x$ be the local coordinate on $X$ with $x \cdot g=v$. Define $y$ on $X$ by $y^{1}=\cos \left(\alpha_{g}\right) x^{1}+\sin \left(\alpha_{g}\right) x^{2}$ and $y^{2}=-\sin \left(\alpha_{g}\right) x^{1}+\cos \left(\alpha_{g}\right) x^{2}$; then $I I^{\prime \prime}$ $=\left(H e^{\lambda^{\prime \prime}}+1\right) d y^{1} \otimes d y^{1}+\left(H e^{\lambda^{\prime \prime}}-1\right) d y^{2} \otimes d y^{2}$ on $X$. We compute

$$
\begin{aligned}
& u^{1}=\cos \left(\alpha_{f}+\alpha_{g}\right)\left(y^{1} \cdot g \cdot f\right)+\sin \left(\alpha_{f}+\alpha_{g}\right)\left(y^{2} \cdot g \cdot f\right) . \\
& u^{2}=-\sin \left(\alpha_{f}+\alpha_{g}\right)\left(y^{1} \cdot g \cdot f\right)+\cos \left(\alpha_{f}+\alpha_{g}\right)\left(y^{2} \cdot g \cdot f\right) .
\end{aligned}
$$

Thus $\alpha_{g \cdot f}=\alpha_{g}+\alpha_{f}$.

Now (ii) and (iv) are immediate.

Let $x \in S_{\Omega}$ and $b \in R$. Choose a local coordinate neighborhood $(U, u)$ of $x$ as in Theorem 3.1. $D=u(U) \subset R^{2}$ is the parameter domain. We define functions $g_{11}(u(z))=g_{22}(u(z))=e^{\lambda(z)}, g_{12}=g_{21}=0, b_{11}(u(z))$ $=H e^{\lambda(z)}+\cos (2 b), b_{22}(u(z))=H e^{\lambda(z)}-\cos (2 b)$ and $b_{12}(u(z))=b_{21}(u(z))$ $=\sin (2 b)$. Then the forms

$$
I_{0}=\sum g_{i j}(u) d u^{i} \otimes d u^{j} \quad \text { and } \quad I I_{0}=\sum b_{i j}(u) d u^{i} \otimes d u^{j}
$$

satisfy the Mainardi-Codazzi equations (4.3). As $S$ satisfies the Gauss equation $a$ priori, and as $I_{0}=I$ and $\operatorname{det}\left(b_{i j}\right)=H^{2} e^{2 \lambda}-1$ as for $S$, now $I_{0}$ and $I I_{0}$ satisfy the Gauss equation. Thus Bonnet's existence theorem says that every $m \in D$ has a neighborhood $V(m) \subset D$ which is parameter domain for a local surface $W \subset R^{3}$ with first and second fundamental forms $\boldsymbol{I}_{0}$ and $I \boldsymbol{I}_{0}$. Let $w$ be the coordinate on $W, m$ $=u(x), V=u^{-1}(V(m))$. Then $h=\left.u^{-1} \cdot u\right|_{v}$ is a diffeomorphism of $V$ on to $W$ such that $h^{*} I_{0}=I_{0}=I$ (so $h$ is an isometry) and $h^{*} I I_{0}=I I_{0}$ (so $\alpha(h)=b$ ). q.e.d.

Theorem 5.1 requires $S_{\Omega}$ to be nonempty. Thus we remark:

5.3. Complement to Theorem 5.1. Let $S$ and $S^{\prime}$ be connected all- 
umbilic surfaces in $R^{3}$. Then any isometry $f: S \rightarrow S^{\prime}$ extends to a rigid motion of $R^{3}$.

For $f$ is a diffeomorphism with $f^{*} I^{\prime}=I$, and we need only check that $f^{*} I I^{\prime}=I I$. An all-umbilic surface is an open subset of a plane or a sphere. In the first case $I I=0$ and $K=0$. In the second case $I I$ $=(1 / r) I$ and $K=1 / r^{2}$ where $r$ is the radius of the sphere. As $f$ preserves Gauss curvature, now $f^{*} I I^{\prime}=I I$. q.e.d.

6. The Gauss Equation. The Gauss equation for a surface $S$ with first fundamental form $e^{\lambda}\left\{d u^{1} \otimes d u^{1}+d u^{2} \otimes d u^{2}\right\}$ says that Gauss curvature is given by ${ }^{5}$

$$
K=-\frac{1}{2} e^{-\lambda} \Delta \lambda, \quad \Delta=\frac{\partial^{2}}{\partial u^{1} \partial u^{1}}+\frac{\partial^{2}}{\partial u^{2} \partial u^{2}} .
$$

Now suppose that $S$ has constant mean curvature $H$ and that our local coordinate neighborhood $(U, u)$ is given by Theorem 3.1. Then $K=H^{2}-e^{-2 \lambda}$, so (6.1) becomes a nonlinear elliptic equation

$$
\Delta \lambda=2\left(e^{-\lambda}-H^{2} e^{\lambda}\right) .
$$

We view this as an equation ${ }^{6}$ for $K=H^{2}-e^{-2 \lambda}$.

We regard (6.2) as a boundary value problem on a disc $D$ of radius $r>0$ in $R^{2}$. Let $b$ be a continuous function on the boundary $\partial D$ of the disc; we look for a solution $\lambda\left(u^{1}, u^{2}\right)$ to (6.2) on $D$, continuous on the closure and with values $b$ on $\partial D$. Let $h\left(u^{1}, u^{2}\right)$ be the harmonic function on $D$ with boundary values $b$. Then we write (6.2) in the form (this defines $F$ )

$$
\Delta \eta=2\left(e^{-(\eta+h)}-H^{2} e^{\eta+h}\right) \equiv F(\eta), \quad \eta=\lambda-h,
$$

and we want a solution $\eta$ on $D$ vanishing on $\partial D$.

Following Courant-Hilbert ([1, Appendix to Chapter 4$])$, such solutions $\eta$ exist provided that certain bounds $c, m$ satisfy $\left(r+r^{2}\right) c m$ $\leqq 1 / 4$. Here $c$ and $m$ are defined as follows. Let $C^{2}(D)$ denote the set of all continuous functions on the closure of $D$ which are twice con-

5 We remark that this has an interesting expression in complex notation. There $z=u^{1}+(-1)^{1 / 2} u^{2}$ is the variable, so $d z=d u^{1}+(-1)^{1 / 2} d u^{2}$ and $d \bar{z}=d u^{1}-(-1)^{1 / 2} d u^{2}$, and the vector fields dual to these forms are $\partial / \partial z=(1 / 2)\left\{\partial / \partial u^{1}-(-1)^{1 / 2} \partial / \partial u^{2}\right\}$ and $\partial / \partial \bar{z}=(1 / 2)\left\{\partial / \partial u^{1}+(-1)^{1 / 2} \partial / \partial u^{2}\right\}$. The exterior derivative $d=d^{\prime}+d^{\prime \prime}$ where by definition $d^{\prime}(f)=(\partial f / \partial z) d z$ and $d^{\prime \prime}(f)=(\partial f / \partial \bar{z}) d \bar{z}$ on functions. In particular $d^{\prime} d^{\prime \prime} f$ $=\frac{1}{4} \Delta f d z \wedge d \bar{z}$. The element of area on $S$ is given by $d A=e^{\lambda} d u^{1} \wedge d u^{2}=(1 / 2)(-1)^{1 / 2} e^{\lambda}$ $\cdot d z \wedge d \bar{z}$. Thus (6.1) can be written in coordinate free form $K d A=-(-1)^{1 / 2} d^{\prime} d^{\prime \prime} \lambda$.

${ }^{6}$ Writing it out in terms of $K$, one obtains $\Delta K=\left\{\left(\partial K / \partial u^{1}\right)^{2}+\left(\partial K / \partial u^{2}\right)^{2}\right\}\left(H^{2}-K\right)$ $+4\left(H^{2}-K\right)^{3 / 2}-4 H^{2}\left(H^{2}-K\right)^{1 / 2}, K<H^{2}$, which is more difficult to study than is (6.2). 
tinuously differentiable in $D$. Then $c$ is specified by

$$
\max \left|\frac{\partial f}{\partial u^{i}}\right| \leqq c r \text { l.u.b. }|\Delta f| \quad \text { for } f \in C^{2}(D),
$$

and $c$ is independent of choice of $r$ or $D$. Define the norm

$$
\|f\|=\max |f|+\sum \max \left|\frac{\partial f}{\partial u^{i}}\right|
$$

on $C^{2}(D)$. Then $m$ is any common bound for all $|F(f)|$ and all $|d F / d f|$ with $\|f\| \leqq 1$. A glance at the form of $F$ in (6.3) shows now that $m$ is any common bound for $2\left(e^{-1-h}+H^{2} e^{1+h}\right)$ and $2\left(e^{1-h}+H^{2} e^{h-1}\right)$. As $h$ achieves its maximum on $\partial D$, now we may take (6.4) $m=\max \left\{2\left(e^{-1-\beta}+H^{2} e^{1+\beta}\right), 2\left(e^{1-\beta}+H^{2} e^{\beta-1}\right)\right\}, \quad \beta=\max |b|$.

In summary, and using the fact that solutions to elliptic equations are analytic,

6.5. Theorem. Let $D$ be a disc of radius $r>0$ in $R^{2}$, let $b$ be a continuous function on $\partial D$, and let $\beta=\max _{\partial D}|b|$. If

$$
\left(r+r^{2}\right)^{-1} \geqq 8 c \cdot \max \left\{e^{-1-\beta}+H^{2} e^{1+\beta}, e^{1-\beta}+H^{2} e^{\beta-1}\right\},
$$

then there exists a continuous function $\lambda$ on the closure of $D$, real analytic on $D$, which satisfies (6.2) and has values $b$ on $\partial D$.

The usual uniqueness condition for an equation $\Delta f=A(f, u)$ for given boundary values is $\partial A / \partial f \geqq 0$. But in our case (6.2) we always have $\partial A / \partial f<0$.

We can now describe the construction of umbilic-free local surfaces of constant mean curvature. Such a surface is specified up to congruence by its first and second fundamental forms, and the condition for two candidates

$$
I=\sum g_{i j} d u^{i} \otimes d u^{j} \quad \text { and } \quad I I=\sum b_{i j} d u^{i} \otimes d u^{j}
$$

to give a surface, is that the $g_{i j}$ and $b_{i j}$ satisfy the Mainardi-Codazzi equations and the Gauss equation.

If $H$ is the mean curvature of the desired surfaces, then Theorem 3.1 allows us to take $I$ and $I I$ in the forms

$$
\begin{aligned}
I= & e^{\lambda}\left\{d u^{1} \otimes d u^{1}+d u^{2} \otimes d u^{2}\right\} \quad \text { and } \\
I I_{t}= & \left\{H e^{\lambda}+\cos (2 t)\right\} d u^{1} \otimes d u^{1}+2 \sin (2 t) d u^{1} d u^{2} \\
& +\left\{H e^{\lambda}-\cos (2 t)\right\} d u^{2} \otimes d u^{2}
\end{aligned}
$$


for $t=0$. For any constant $t$, the Mainardi-Codazzi equations (4.3) are satisfied for $I$ and $I I_{t}$, and the Gauss equation (6.2) is

$$
\Delta \lambda=2\left(e^{-\lambda}-H^{2} e^{\lambda}\right), \quad K=H^{2}-e^{-2 \lambda} .
$$

Theorem 6.5 gives the existence of many solutions. Given a local solution $\lambda$, there corresponds a well defined congruence class of local surfaces $S_{\lambda, t}$ with $I$ as specified and $I I=I I_{t}$; Theorem 5.1 shows that the natural map $f_{z, t}: S_{\lambda, s} \rightarrow S_{\lambda, t}$ is an isometry and $\alpha\left(f_{s, t}\right)=t-s$.

\section{REFERENCES}

1. R. Courant, Methods of mathematical physics, II: Partial differential equations, Interscience, New York, 1962.

2. H. Hopf, Selected topics in differential geometry in the large, Notes by T. Klotz, New York University, New York, 1955.

3. J. A. Wolf, Exotic metrics on immersed surfaces, Proc. Amer. Math. Soc. 17 (1966), 871-877.

The Institute for Advanced Study and University of California, Berkeley

${ }^{7} f_{s, t}$ is given by $u^{i}\left(f_{s, t}(x)\right)=u^{i}(x)$. 